\title{
A FUZZY SYSTEM FOR MULTIOBJECTIVE PROBLEMS
}

A Case Study in NP-Hard Problems

M. R. Gholamian, S. M. T. Fatemi Ghomi and M. Ghazanfari

Department of Industrial Engineering, Amirkabir University of Technology, $424 \mathrm{Hafez}$ Avenue, Tehran, Iran, \{Gholamian, Fatemi\}@aut.ac.ir

Department of Industrial Engineering, Iran University of Science and Technology, Narmak 16844, Tehran, Iran, mehdi@iust.ac.ir.

Abstract: In this study, an intelligent fuzzy system is used instead of mathematical models. The main core of the system is fuzzy rule base which maps decision space $(Z)$ to solution space $(X)$. The system is designed on noninferior region and gives a big picture of this region in the pattern of fuzzy rules. In addition, numerical examples of well-known NP-hard problems (i.e. multiobjective traveling salesman problem and multiobjective knapsack problem) are provided to clarify the accuracy of developed system.

Key words: Multiobjective decision making, Fuzzy Systems, NP-hard Problems

\section{INTRODUCTION}

A multiobjective problem is generally defined as follows [21]:

$$
\begin{array}{cc}
\operatorname{Max} \mathbf{F}(\mathbf{x})=\left(\mathrm{f}_{\mathrm{l}}(\mathbf{x}), \mathrm{f}_{2}(\mathbf{x}) \ldots \mathrm{f}_{\mathrm{P}}(\mathbf{x})\right) \\
\mathrm{g}_{\mathrm{i}}(\mathbf{x}) \leq 0 \quad \mathrm{i}=1,2, \ldots, \mathrm{m} \\
\mathrm{h}_{\mathrm{j}}(\mathbf{x})=0 & \mathrm{j}=1,2, \ldots, \mathrm{m}^{\prime} \\
\mathbf{x} \geq 0 &
\end{array}
$$

where $\mathbf{x}$ is the vector with $\mathbf{n}$ decision variables. $\mathbf{f}: R^{n} \rightarrow R^{P}$ are real valued functions which may be non-differentiable, discontinuous, non-smooth and generally difficult to express mathematically. On the other hand, $g: R^{n} \rightarrow R^{m}$ 
and $\mathbf{h}: \mathrm{R}^{\mathrm{n}} \rightarrow \mathrm{R}^{\mathrm{In}}$ are also real valued functions. For simplicity, in this paper $f_{1}(\mathbf{x}), f_{2}(\mathbf{x}) \ldots f_{\mathrm{p}}(\mathbf{x})$ are shown as $\mathrm{z}_{1}, \mathrm{z}_{2} \ldots \mathrm{z}_{\mathrm{p}}$ respectively.

In principle, multiobjective problems are very different than the single objective problems. In single objective problems, one attempts to obtain the best design or decision, which is usually the global minimum or maximum depending on the problem definition. In the case of multiobjective, there may not exist one solution which is the best (global minimum or maximum) with respect to all objectives; instead there exists a set of solutions which are superior respect to the rest of solutions in solution space when all objectives are considered but are inferior respect to other solutions in the space in one or more objectives. These tradeoff solutions are known as noninferior points [18]. In other word $\mathbf{x}$ is a noninferior solution if and only if:

$\neg \exists \mathrm{x}^{\prime} \in \mathrm{R}^{\mathrm{n}}: \forall \mathrm{i} \in\{1, \ldots, \mathrm{p}\} \mathrm{F}_{\mathrm{i}}\left(\mathrm{x}^{\prime}\right) \geq \mathrm{F}_{\mathrm{i}}(\mathrm{x}) \wedge \exists \mathrm{i} \in\{1, \ldots, \mathrm{p}\} \mathrm{F}_{\mathrm{i}}\left(\mathrm{x}^{\prime}\right)>\mathrm{F}_{\mathrm{i}}(\mathrm{x})$

To find such solutions, many mathematical and stochastic based methods are developed. In particular, the recent works are concentrated on multiobjective evolutionary algorithms [4]. But these approaches would not support unstructured or semi-structured problems. The approaches are founded on specified mathematical models and hence vagueness, imprecision and incompleteness can not be supported with these approaches [19].

In contrast, the knowledge based systems are traditionally used in such ill-structured cases of real world problems [10]. Specially, various rule based systems are developed in a wide range of ill-structured optimization problems. However, in multiobjective problems the applications are restricted to marginal or auxiliary of other implementation methods.

The systems are used along with Meta-heuristics and even classical approaches. For example Nabrzski and Weglarz [15] introduced a rule based system aside with a Tabu search method to control Tabu list, choose neighbors, define aspiration level and change attributes. EESA [13] and PAMUCII [5] are other samples of such rule based applications. In applications with classical methods for example Poulos et al. [16] used fuzzy rule based system to control incremental changes of weights in a weighting method for a warehouse multiobjective problem. As another work, Agrell et al. [1] developed a rule based decision support system aside with Tchebycheff interactive method to provide an agro-ecological and economic assessment of various types of land uses. Also, Rasmy et al. [17] developed fuzzy expert system to extract priorities and aspiration levels based on 
linguistic preferences and then convert multiobjective problems to equivalent goal programming model.

Fuzzy rule bases are also used to extract unstructured objective functions of decision maker [2]. The fuzzy rules are developed with linguistic variables in antecedent and linguistic objectives in consequents and then Tsukamoto's fuzzy reasoning method is used to determine crisp mathematical structure of objective functions. Similarly, Sugeno's fuzzy reasoning method with fuzzy decision making method are used as MANFIS network, which is an extension of the ANFIS network for fuzzy multiobjective problems to extract related single objective model and then this model is optimized via genetic algorithm [3].

As shown in all applications, the rule bases are used with marginal roles. In contrast, in this study the rule bases are used with an original role. Specially in this study, a system constituting fuzzy rule-base is used [8]. Since decision maker interface is goal space $(Z)$ and the system must produce the solutions, system inferencing is developed as $Z$ to $X$ mapping and fuzzy logic is used to support uncertainty [11]. Meanwhile, since the knowledge base is generated in noninferior region, the system can be used as an analyzer for decision maker and he/she can analyze various levels of goals and observe related solutions. Then the system not only searches the noninferior region but also helps analyzer to make the best decisions in this region [7].

The rest of the paper is organized as follows. Section 2 introduces structure of the system and describes the steps of system development. To clarify the performance of developed system, two well-known numerical examples (i.e. multiobjective TSP problem and multiobjective knapsack problem) are brought in section 3. The fuzzy rule-based system is developed for each example and results are explained clearly. Finally section 4 is devoted to conclusions and recommendations for future studies.

\section{SYSTEM STRUCTURE}

The system structure developed in this paper is more attended to illstructured problems; ones which traditional methods can not acquit them. Originally, the system vision is different. In this vision instead of attending to the elements of the problem and generating complex mathematical models, concentration is made on global rules which the problem is dominated and 
controlled with them. This is the reason of using rule bases in the structure of the system.

On the other hand, most of real problems are defined in uncertain conditions with inadequate information. This vagueness and imprecision is supported with fuzzy logic [6] and hence fuzzy rule base is suggested for the system.

In designing fuzzy rule-based systems, if familiar experts and elites exist, the rules can be extracted of them. But this operation may be bias of these specialists which will not be representative of real noninferior region.

Another way may be usage of historical data. When set of historical data exists, the information can be evaluated to extract noninferior subset as an estimation of real noninferior population. This operation can be performed with simple procedure developed for extraction of real noninferior set. The outline of this procedure is as follows:

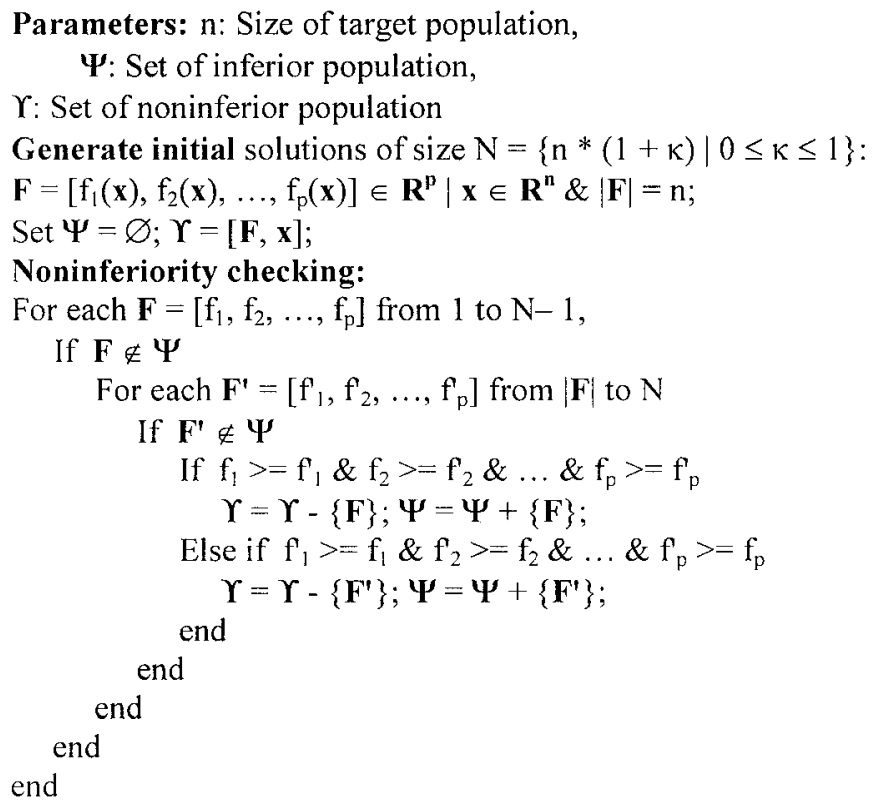

In the next step, the resulted noninferior points are used in designing of fuzzy rule base. Rules are defined based on Gaussian membership functions; because in general cases, most of the membership functions are converged to this typical smooth one [12]: 
$\mu_{1}([\mathbf{F}(\mathbf{x}), \mathbf{x}])=\operatorname{EXP}\left(\frac{-\left([\mathbf{F}(\mathbf{x}), \mathbf{x}]-\left[\mathbf{F}\left(\mathbf{x}_{i}\right), \mathbf{x}_{\mathrm{i}}\right]\right)^{2}}{2 \sigma_{[F(x), \mathbf{x}]}^{2}}\right),[\mathbf{F}(\mathbf{x}), \mathbf{x}] \in \mathrm{R}^{\mathrm{p}+\mathrm{n}}, \mathrm{i}=1,2, \ldots \mathrm{n}$

where $\mathrm{n}$ is number of noninferior points and $\sigma_{[\mathrm{F}(\mathbf{x}), \mathbf{x}]}$ is defined as follows:

$\sigma_{[F(x), x]}=\left(\frac{\sum_{i}\left[\mathbf{F}\left(\mathbf{x}_{\mathbf{i}}\right), \mathbf{x}_{\mathbf{i}}\right]^{2} \mu_{i}([\mathbf{F}(\mathbf{x}), \mathbf{x}])}{n}-\left(\frac{\sum_{i}\left[\mathbf{F}\left(\mathbf{x}_{\mathrm{i}}\right), \mathbf{x}_{\mathbf{i}}\right] \mu_{i}([\mathbf{F}(\mathbf{x}), \mathbf{x}])}{n}\right)^{2}\right)^{\frac{1}{2}}$

Then, the fuzzy rules $\mathrm{R}: \mathrm{A} \rightarrow \mathrm{B}$ are defined as follows [14]:

$$
\begin{aligned}
& A_{j}=\int_{f(x) \in R^{\prime}}\left(\left(\mu_{j}(f(x)) \mid f(x)\right) \quad j=1,2, \ldots, p\right. \\
& B_{i}=\int_{x \in R^{n}}\left(\mu_{i}(x) \mid \mathbf{x}\right) \quad i=1,2, \ldots, n
\end{aligned}
$$

Now, Mamdani inferencing system [12] is used on developed rules to constitute the fuzzy rule base. Let $\mathbf{z}$ as the input vector of decision space, then the rule $R_{r}$ is fired as follows:

$\tau_{\mathrm{r}}=\widehat{\mathrm{j}}_{\mathrm{j}=1}^{\mathrm{p}} \mu_{\mathrm{j}}^{\mathrm{r}_{\mathrm{j}}}\left(\mathrm{z}_{\mathrm{j}}\right) \mid \mathrm{A}_{\mathrm{j}}^{\mathrm{r}_{\mathrm{j}}} \& \mathrm{~A}_{\mathrm{j}}^{\mathrm{r}_{j}}=\left(\int_{\mathbf{z} \in \mathrm{R}^{r}} \mu_{\mathrm{j}}^{\mathrm{r}_{\mathrm{j}}}(\mathbf{z}) \mid \mathbf{z}\right) \subset \mathrm{A}_{j}$

and then, the inferred consequences are obtained as follows:

$\mu_{r}{ }^{\text {Cons }}(\mathbf{x})=\tau_{r} \wedge \mu_{i}^{r_{i}}\left(x_{i}\right) \mid B_{i}^{r_{i}} \& B_{i}^{r_{i}}=\left(\int_{x \in R^{n}} \mu_{i}^{r_{i}}(x) \mid \mathbf{x}\right) \subset B_{i}$

Finally, the aggregation is performed as follows:

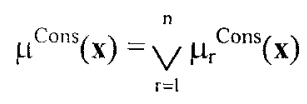

The t-norm and t-conorm operators either may be simple, such as standard, product, drastic and bounded operators or may be parametric such as Yager class, Schweizer \& Sklar classes, Hamacher class and Dubois class [22].

Finally, the solutions must be defuzzified to obtain crisp trading values. In this part also parametric and non-parametric methods can be used. 
Specially, SLIDE generalized defuzzification method [20] is applied as follows:

$$
x^{*}=\frac{(1-\beta) \int_{i \in L} x \mu_{i}^{\alpha}(x) d x+\beta \int_{i \in H} x \mu_{i}^{\alpha}(x) d x}{(1-\beta) \int_{i \in L} \mu_{i}^{\alpha}(x) d x+\beta \int_{i \in H} \mu_{i}^{\alpha}(x) d x} \quad \alpha \in\left[0, \operatorname{Height}\left(\mu_{i}(x)\right)\right], \beta \in[0,1]
$$

where $L=\left\{\mathrm{i} \mid \mu\left(\mathrm{x}_{\mathrm{i}}\right)<\alpha\right\}$ and $\mathrm{H}=\left\{\mathrm{i} \mid \mu\left(\mathrm{x}_{\mathrm{i}}\right) \geq \alpha\right\}$.

It should be attended that most of noninferior points are not sensible for decision maker and it is necessary to focus on the highlighted areas; the work which is not performed with traditional methods.

These sensible points are supported in the system at arrival to fuzzy rulebased system. In fact an activated area is given to the system instead of entire area. Focusing in activated area may be performed by decision maker. Generally managers and specialists continuously impact with various objectives of the firm such as price, quality, and delivery and hence can comfortably explain critical interval of changes in each objective [9]. This is one of the reasons that $Z$ to $X$ mapping is suggested for the system. In fact, the arrival to the system is what the decision maker knows ( $z$ ) and the departure of the system is what the decision maker wants $(\mathrm{x})$.

Now, suppose the situation that activation area is not sensible for decision maker; then this area must be obtained by the system. In existence of mathematical structure following payoff table can be produced with the optimal solutions.

Table 1: Payoff table of optimal solutions

\begin{tabular}{c|cccc} 
& $\mathrm{f}_{1}$ & $\mathrm{f}_{2}$ & $\ldots$ & $\mathrm{f}_{\mathrm{p}}$ \\
\hline $\mathrm{x}_{1}{ }^{*}$ & $\mathrm{f}_{1}\left(\mathrm{x}_{1}{ }^{*}\right)$ & $\mathrm{f}_{2}\left(\mathrm{x}_{1}{ }^{*}\right)$ & $\ldots$ & $\mathrm{f}_{\mathrm{p}}\left(\mathrm{x}_{1}{ }^{*}\right)$ \\
$\mathrm{x}_{2}{ }^{*}$ & $\mathrm{f}_{1}\left(\mathrm{x}_{2}{ }^{*}\right)$ & $\mathrm{f}_{2}\left(\mathrm{x}_{2}{ }^{*}\right)$ & $\ldots$ & $\mathrm{f}_{\mathrm{p}}\left(\mathrm{x}_{2}{ }^{*}\right)$ \\
$\vdots$ & $\vdots$ & $\vdots$ & $\ldots$ & $\vdots$ \\
$\mathrm{x}_{\mathrm{p}}{ }^{*}$ & $\mathrm{f}_{1}\left(\mathrm{x}_{\mathrm{p}}{ }^{*}\right)$ & $\mathrm{f}_{2}\left(\mathrm{x}_{\mathrm{p}}{ }^{*}\right)$ & $\ldots$ & $\mathrm{f}_{\mathrm{p}}\left(\mathrm{x}_{\mathrm{p}}{ }^{*}\right)$
\end{tabular}

In above table, the positive ideal and negative-ideal solutions can be identified as follows:

$\mathrm{A}^{\prime}=\left\{\mathrm{V}_{j}^{+} \mid \mathrm{V}_{\mathrm{j}}^{+}=\operatorname{Max}_{\mathrm{i}=1}^{p} \frac{\mathrm{f}_{j}\left(x_{\mathrm{i}}^{*}\right)}{\left\|\mathrm{f}_{\mathrm{j}}\left(x_{\mathrm{i}}^{*}\right)\right\|^{\kappa}}\right\} \quad \mathrm{A}^{-}=\left\{\mathrm{V}_{\mathrm{j}}^{-} \mid \mathrm{V}_{j}^{-}=\operatorname{Min}_{\mathrm{j}=1}^{p} \frac{\mathrm{f}_{j}\left(x_{i}^{*}\right)}{\left\|\mathrm{f}_{\mathrm{j}}\left(x_{\mathrm{i}}^{*}\right)\right\|^{\kappa}}\right\}$ 
Then, similar to TOPSIS method, the following index gives decision maker an idea to determine the boundaries of activated areas:

$$
I_{i}^{*}=\left\{\frac{\left\|V_{j i}-V_{j}^{+}\right\|^{\kappa}}{\left\|V_{j i}-V_{j}^{+}\right\|^{k}+\left\|V_{j i}-V_{j}^{-}\right\|^{\kappa}} \mid V_{j i}=\frac{f_{j}\left(x_{i}^{*}\right)}{\left\|f_{j}\left(x_{i}^{*}\right)\right\|^{k}}\right\}
$$

When activation area is obtained, this area is atomized to set of grid points by splitting the intervals and producing ordered vector of goal values. This is the set of points which are given to fuzzy rule-based system as activation points. The generation of these points is integrated in the system as "Z Generator" gate.

Finally, the output of the system is projected in decision space in order to evaluate the resultant performance of the system

\section{NUMERICAL EXAMPLES}

In this section, to clarify the performance of fuzzy rule-based system, two well-known problems of multiobjective traveling salesman problem (MOTSP) and multiobjective knapsack problem (MOKP) are suggested and the system is developed for each of them. In this study, programs are developed with MATALB software on the Pentium personal computer.

\subsection{Multiobjective TSP problem (MOTSP)}

MOTSP is formulated in the following way. Let $U=\{1,2 \ldots n\}$ be the set of cities and $\mathrm{C}^{(1)}, \mathrm{C}^{(2)}, \ldots, \mathrm{C}^{(\mathrm{p})}$ the distance matrices of cities. Also let $\mathbf{X}=$ $\left\{x_{i j} \mid \mathrm{i}, j \in U\right\}$ be the binary matrix of decision variables. Then the multiobjective TSP problem is formulated as follows:

$\operatorname{Min} \mathbf{F}(\mathbf{X})=\left(\mathbf{C}^{(1)} * \mathbf{X}, \mathbf{C}^{(2) *} \mathbf{X} \ldots \mathbf{C}^{(\mathbf{p}) * \mathbf{X}}\right)$

Subject to:

$$
\begin{aligned}
& \left\|\mathbf{X}_{\mathrm{i}}\right\|_{1} \&\left\|\mathbf{X}_{\mathrm{j}}\right\|_{1}=1 \quad \forall \mathrm{i}, \mathrm{j} \in \mathrm{U} \\
& \sum_{i \in V} \sum_{i \in l \backslash V} x_{i j} \geq 1 \quad \forall \mathrm{V} \subset \mathrm{U}(\mathrm{V} \neq \varnothing, \mathrm{V} \neq \mathrm{U}) \\
& \mathrm{x}_{\mathrm{ij}}=[0,1] \quad \forall \mathrm{i}, \mathrm{j} \in \mathrm{U}
\end{aligned}
$$

Obviously, while single objective of this problem is NP-hard, the multiobjective ones, is also NP-Hard. The MOTSP solutions can be 
represented as a sequence of cities with related objective values. Let $\mathrm{S}$ be solution of the problem, then $\mathrm{S}$ can be represented as follows:

$S=\left[f_{1}\left(U^{O}\right), f_{2}\left(U^{O}\right), \ldots, f_{p}\left(U^{\circ}\right), U^{O}\right]$

where $U^{O}$ is the ordered set of $U$ based on the sequence of cities. Since only $\mathrm{n}$ ! sequences of the cities can be generated, $\mathrm{n}$ ! S solutions can be produced.

In this study, a bi-objective model is developed with opposite goals (Max profit and Min Risk) for 8 cities problem (because of restriction in illustration, 8 cities problem is selected). $\mathbf{C}^{(1)}$ and $\mathbf{C}^{(2)}$ are generated randomly with uniform distribution in the ranges of $[0,100]$ and $[0,10]$ respectively.

As the beginning, $5000 \mathrm{~S}$ solutions are generated. This is approximately $\% 10$ of total solutions. The operation is performed in the time $2.344^{\mathrm{s}}$. Then noninferior solutions are extracted using noninferior procedure. Interestingly from 5000 random points only 8 noninferior solutions are extracted in the time less than $0.01^{\mathrm{s}}$. In the next stage, fuzzy rules are developed. Membership functions are selected based on Gaussian functions with uniform variance on the activation area. The activation area of outputs is selected in the range $[0,8]$ to support city selection (The output must be rounded to obtain selected cities). The activation area of inputs is obtained as $240 \leq z_{1} \leq 360$ and $1 \leq z_{2} \leq 2.5$. The operators are selected by default in the Mamdani system and finally the following rule base is obtained:
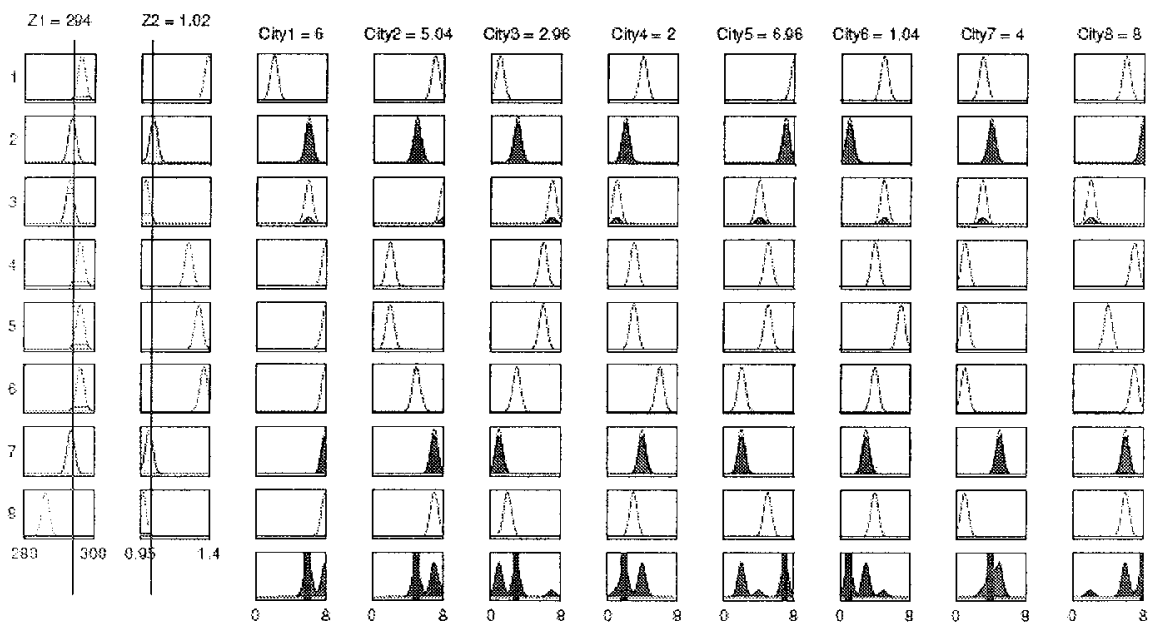

Figure 1. Fuzzy rule base of MOTSP problem 
This rule base is constructed on noninferior region, and then changing the goal levels $\left(z_{1}\right.$ and $\left.z_{2}\right)$ by simply sliding the red lines, caused other noninferior path solutions. In fact, the system let decision maker to analyze various solutions based on various decisional levels. While in traditional methods even in Meta heuristic ones, this analyzing needs a lot of repetitive calculations with huge time consumption, in this system decision maker can analyze various paths with various profit and distance and then select the pleasure ones easily and simply.

As an additional work, to examine the performance of developed system, the activated area (i.e. $240 \leq z_{1} \leq 360$ and $1 \leq z_{2} \leq 2.5$ ) is divided to the grids with the rates 1 and 0.1 respectively. Then each couple points of grid region is given to the system as input values $\left(z_{1}\right.$ and $\left.z_{2}\right)$ and on the other hand, the system generates outputs as a sequence of the cities immediately.

After rounding the solutions, a validation test is performed on the sequences and accordingly, 1831 solutions are accepted from 1936 ones (i.e. $\% 94.58$ of solutions). The checking process is performed in only $1.762^{\mathrm{s}}$.

The accepted solutions are projected in decisional space. The result was wonderful. While input space is complete grid points, the output projection space is quite adapted to the noninferior region; such as shown in the following Figure.
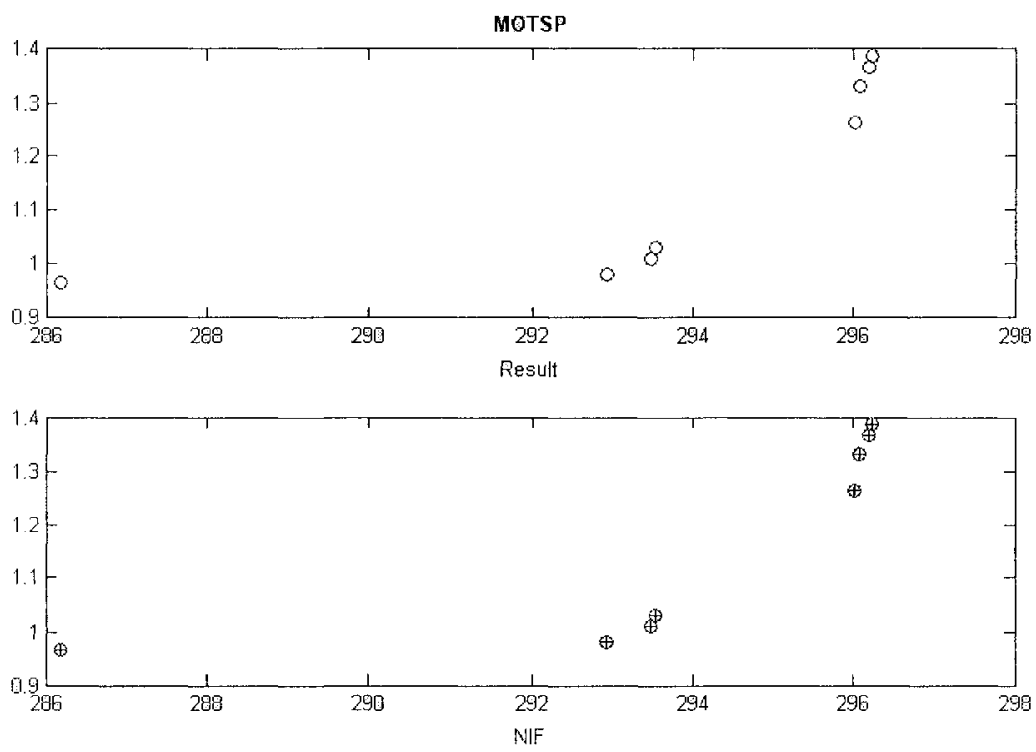

Figure 2. Comparison of resulted points and noninferior points in MOTSP problem 


\subsection{Multiobjective knapsack problem (MOKP)}

MOKP is formulated in the following way. Let $\mathbf{g}$ be vector of element weights and $\mathbf{c}^{(1)}, \mathbf{c}^{(2)}, \ldots, \mathbf{c}^{(p)}$ vectors of element values. Then multiobjective knapsack problem is formulated as follows:

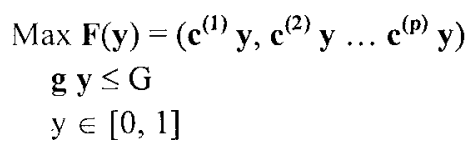

where $\mathrm{G}$ is a scalar value corresponding to the knapsack size and $y$ is the vector of variables.

Again, in this example a bi-objective model with opposite goals (Max confidence, Min cost) and 8 items is developed. The weights are generated randomly in the range $[0,1]$ and $G$ is set to $G=\|g\|_{1} / 2$. The vectors of element values are generated randomly; but in different ranges. $c^{(1)}$ is generated in the range $[0,1]$ while $\mathrm{c}^{(2)}$ is generated in $[0,1000]$.

Similar to previous example, the work is begun with generation of random binary vector $(y)$. Since the model is mono-constraint, the feasibility of random solutions is checked and only feasible ones are used. In this fashion, 1000 random feasible solutions are generated at $0.07^{\mathrm{s}}$. Then, using described noninferior procedure, 8 noninferior solutions are extracted in the time less than $0.01^{\mathrm{s}}$. The obtained noninferior points are used in rule construction.
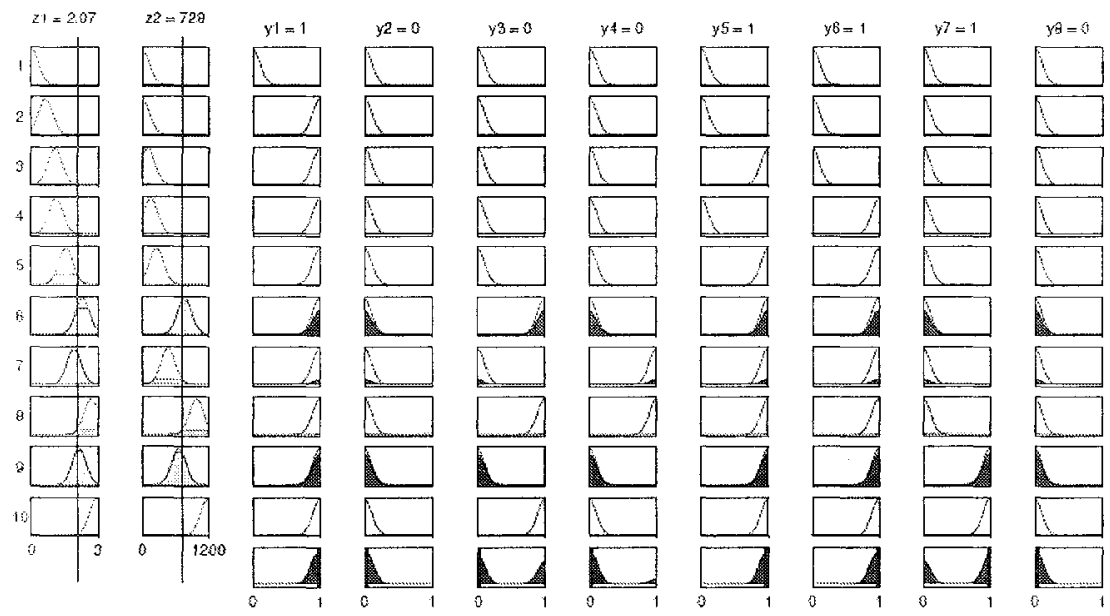

Figure 3. Fuzzy rule base of MOKP problem 
Again Mamdani system is used with default operators and membership functions. While outputs range are obviously $[0,1]$, the inputs range are obtained by plotting initial feasible solutions as $0 \leq z_{1} \leq 3.5$ and $0 \leq z_{2} \leq$ 4000 . Finally the generated rule base is obtained as shown in Figure 3.

Such as shown, the outputs are always binary values and feasible in noninferior region. Then the system can be used as an analyzer of various goal levels and the related solutions. In fact, decision maker can select various subsets of elements based on the desired composition of goals in noninferior region without any additional computation efforts.

Similar to previous example, to show the performance of the system, a grid point set of activation area (i.e. $0 \leq z_{1} \leq 3.5$ and $0 \leq z_{2} \leq 3500$ ) is generated with step sizes 0.1 and 10 respectively and couple points $\left(z_{1}, z_{2}\right)$ are given to the system. The outputs are produced immediately as binary vectors. Then, the feasibility test is performed on the solutions and all of 12636 solutions are accepted. The solutions are projected in decisional space as illustrated in the following figure. Such as shown, the solutions fall in the noninferior boundaries; while the inputs of the system were couple points from all of activation area.
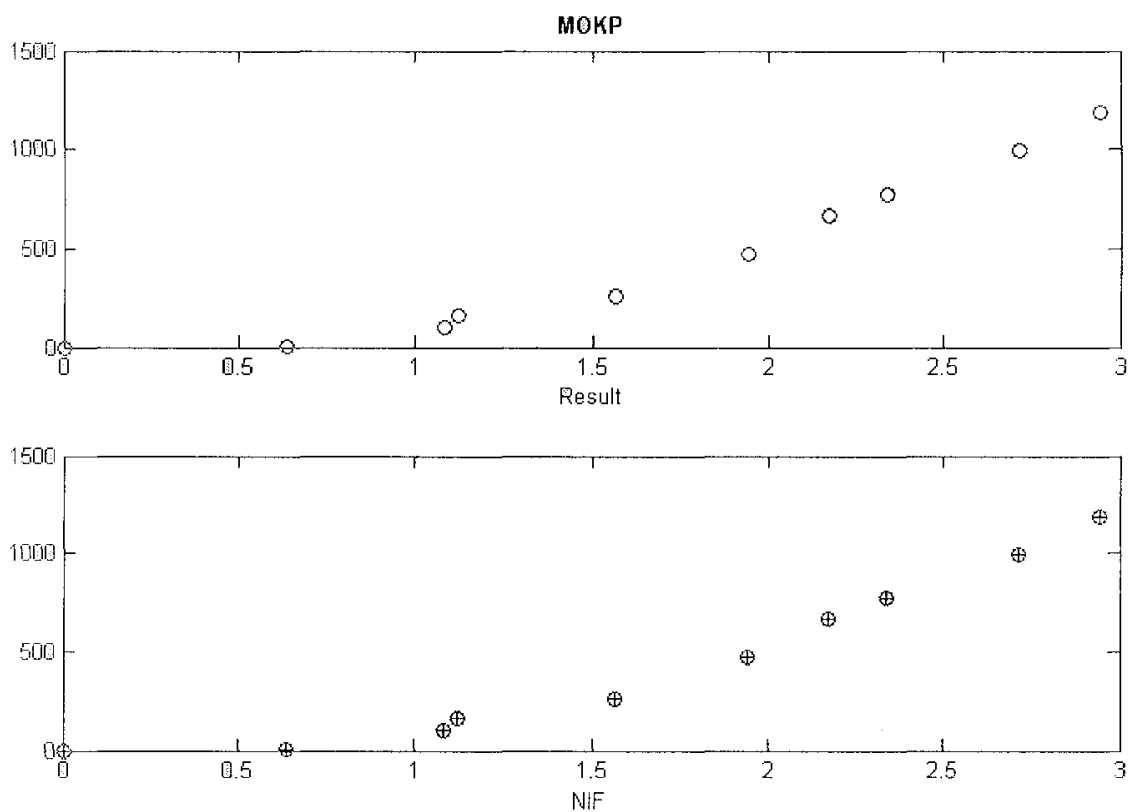

Figure 4. Comparison of resulted points and noninferior points in MOKP problem 


\section{FUTURE STUDIES}

The system structure can also be suggested as another aspect of future study. Some of real problems have not been constructed in predefined domain theory and would not be explained with rules. In these situations, some features are defined for the problem and then an analogical inferencing system along with historical case base is used as case-based reasoning (CBR) system instead of rule-based reasoning (RBR) system. The system is more suitable for ill-structured problems and the problems which can not be represented with mathematical models.

\section{REFERENCES}

[1] Agrell PJ, et al. Interactive multiobjective agro-ecological land use planning: The Bungoma region in Kenya. European Journal of Operational Research, 2004, 158(1): 194 217

[2] Carlsson C, Fuller R. Multiobjective linguistic optimization. Fuzzy Sets and Systems, 2000, 115: 5 10

[3] Cheng CB, et al. Neuro-fuzzy and genetic algorithm in multiple response optimization. Computers and Mathematics with Applications, 2002, 44(12): 1503 1514

[4] Coello C, et al. Evolutionary Algorithms for Solving Multi-Objective Problems. Kluwer, New York, 2002

[5] Coelho RF, Bouillard P. PAMUC II for multicriteria optimization of mechanical designs with expert rules. In: Proceedings of the Congress on Evolutionary Computation (CEC2004). Portland, 2004, 1: 17 22

[6] Ghazanfari M, Kazemi Z. The principle of expert systems. IUST Publisher, Tehran, 2003

[7] Gholamian, MR, et al. A systematic design of multiobjective problem solutions with fuzzy rule based systems. In: Proceedings of the 6th International Multi-Objective Programming and Goal Programming conference (MOPGP'04). Hammamet, Tunisia, 2004

[8] Gholamian MR, Fatemi Ghomi SMT. A hybrid intelligent system for multiobjective decision making problem. In: Proceedings of the 33rd International Conference on Computers and Industrial Engineering (C\&IE2004). Jeju, Korea, 2004

19] Gholamian MR, Fatemi Ghomi SMT. A hybrid system oscillator for multiobjective supplier selection problem. In: Proceedings of the XVII-th International Conference on Multiple Criteria Decision Making. Whistler, British Columbia, Canada, 2004

[10] Gholamian MR, Fatemi Ghomi SMT. An empirical research in intelligent manufacturing: a frame based representation of AI usages in manufacturing aspects. In: proceedings of the 6th IFIP International Conference on Information Technology for Balanced Automation Systems in Manufacturing and Services (BASYS'04). Vienna, Austria, 2004

[11] Gholamian MR, et al. A hybrid system for multiobjective marketing problems- A case study in crude oil markets. Engineering Applications of Artificial Intelligence, 2005, 18(4): 495 509.

[12] Jang J-SR, Sun C-T. Neuro-Fuzzy and Soft Computing: A Computational Approach to Learning and Machine Intelligence. Prentice Hall, New Jersey, 1997 
[13] Jwo WS, et al. Hybrid expert system and simulated annealing approach to optimal reactive power planning. In: IEE Proceedings of Generation, Transmission and Distribution. 1995, 142(4): 381-385.

[14] Kosko B. Fuzzy engineering. Prentice Hall, New Jersey, 1997

[15] Nabrzyski J, Weglarz J. On an expert system with multicriteria Tabu search for multiobjective project scheduling. In: Proceedings of the 6th International Conference on Emerging Technologies and Factory Automation. 1997, 287 -291.

[16] Poulos PN, et al. A Pareto optimal genetic algorithm for warehouse multi-objective optimization. Engineering Applications of Artificial Intelligence, 2001, 14: 737 749

[17] Rasmy MH, et al. An expert system for multiobjective decision making: application of fuzzy linguistic preferences and goal programming. Fuzzy Sets and Systems, 2002, 127 : 209 220.

[18] Steuer PE, Multiple criteria optimization: theory, computation and application. John Wiley \& Sons, New York, 1986

[19] Van Veldhauizen D, Lamont GB. Multiobjective evolutionary algorithms: analyzing the state-of-the-art. Evolutionary Computation, 2000, 8(2): 125 147.

[20] Yager RR, Zadeh LA. Fuzzy sets, neural networks and soft computing. Van Nostrand Reinhold, New York, 1994

[21] Zeleny M. Multiple criteria decision making: Eight concepts of optimality. Human Systems Management, 1998, 17: 97 107

[22] Zimmmermann HJ. Fuzzy set theory and its applications. 3rd edn. Kluwer, Boston, 1996 